\title{
Exogenous Carbon Magnifies Mycorrhizal Effects on Growth Behaviour and Sucrose Metabolism in Trifoliate Orange
}

\author{
Li TIAN ${ }^{1,2}$, Yan $\mathrm{LI}^{1}$, Qiang-Sheng WU $\mathrm{U}^{1,2,3 *}$ \\ ${ }^{1}$ Yangtze University, College of Horticulture and Gardening, Jingzhou, Hubei 434025, China; 18255065993@163.com; 516330488@qq.com; \\ wuqiangsh@163.com (*correspondingauthor) \\ ${ }^{2}$ Yangtze University, Institute of Root Biology, Jingzhou, Hubei 434025, China \\ ${ }^{3}$ University of Hradec Kralove, Faculty of Science, Department of Chemistry, Hradec Kralove, 50003, Czech Republic
}

\begin{abstract}
Arbuscular mycorrhizas (AMs) need the carbohydrates from host plants for its growth, whereas it is not clear whether exogenous carbon affects mycorrhizal roles. A two-chambered rootbox was divided into root + hyphae chamber and hyphae chamber (free of roots) by $37-\mu \mathrm{m}$ nylon mesh, in which trifoliate orange (Poncirus trifoliata) seedlings and Funneliformis mosseae were applied into root + hyphae chamber, and exogenous $40 \mathrm{mmol} / \mathrm{L}$ fructose, glucose and sucrose was applied to hyphae chamber. Application of exogenous sugars dramatically elevated root mycorrhizal colonization. Sole arbuscular mycorrhizal fungi (AMF) inoculation significantly promoted plant growth and root morphology than non-AMF treatment. Mycorrhiza-improved plant growth and root modification could be enlarged by exogenous carbon, especially fructose. Exogenous carbon markedly increased root fructose, glucose and sucrose accumulation in mycorrhizal plants, especially sucrose. Exogenous fructose significantly reduced leaf and root sucrose synthase (SS) activity in synthesis direction and increased them in cleavage direction in AMF seedlings. Exogenous glucose and sucrose heavily elevated root SS activity of mycorrhizal seedlings in synthesis and cleavage direction and reduced leaf SS activity in synthesis direction. Leaf acid invertase (AI) and neutral invertase (NI) activities of mycorrhizal seedlings were decreased by exogenous carbon, except sucrose in NI. Exogenous fructose significantly increased root AI and NI activity in mycorrhizal plants. These results implied that mycorrhizal inoculation represented positive effects on plant growth, root morphology, and sucrose metabolism of trifoliate orange, which could be magnified further by exogenous carbon, especially fructose.
\end{abstract}

Keywords: arbuscular mycorrhizal fungi; citrus, fructose; sucrose invertase; sucrose synthase

\section{Introduction}

Plant growth and development are tightly controlled by environmental conditions, which influence the availability of carbohydrates (O'Hara et al., 2013). Arbuscular mycorrhizal fungi (AMF), a kind of soil microorganisms, can build symbiotic associations with approximately $80 \%$ of terrestrial plants to form arbuscular mycorrhizas (AMs). AMs help the host plant to absorb both water and nutrients as well as enhance the tolerance of biotic and abiotic stresses, in exchange for acquiring 4-20\% additional carbohydrates from the host plant (Gadkar et al., 2001; Wu et al., 2011). In this regard, root sucrose needs to be broken down into hexoses in the presence of sucrose-cleaved enzymes, because AMF could only take in micro-molecules (Bago et al., 2003). Previous studies had shown that AMF colonization induced significant changes in activities of sucrose-cleaving enzymes (Schubert et al., 2004; Wu et al., 2013).

Carbon (C) is one of the vital substances of plant cytoskeleton, and plays an irreplaceable role in the photosynthesis process. He et al. (2006) reported that soluble sugar in tomato was positively related to root mycorrhizal colonization. Bago et al. (2000, 2002, 2003) proposed the $\mathrm{C}$ metabolism in AM symbiosis: organic nutrients are transferred from host plants to internal hyphae in the form of hexose, synthesized into glycogen and triacylglycerol through a series of metabolic pathways, transferred from internal hyphae to external hyphae, releasing energy for the growth and development of AMs. However, recent researches showed that fatty acids were the major source of organic $\mathrm{C}$ delivered from the host plants to the AM fungus (Jiang et al., 2017; Luginbuehl et al., 2017). 
366

A study in the past showed that in beech plants, mycorrhizas principally accumulated trehalose and glycogen from exogenous glucose and trehalose, and mannitol from fructose and mannitol (Lewis and Harley, 1965). Jin et al. (2011) observed that exogenous glucose supply to the AM fungal spores produced a significant enhancement in the absorption of exogenous $\mathrm{N}$ sources, resulting in more than 3 time free amino acid production. Such results indicated the important roles of exogenous $\mathrm{C}$ in mycorrhizal development.

Trifoliate orange [Poncirus trifoliata (L.) Raf.], a close Citrus species, heavily depends on AMs to assimilate nutrients from soils based on its shallow root system with few or no root hairs (Zobel et al., 2006). In this background, the present work evaluated the effects of Funneliformismosseae and exogenous carbohydrates on plant growth, root morphology, carbohydrate concentrations, sucrose synthase activity, and sucrose invertase activity of trifoliate orange seedlings.

\section{Materials and Methods}

\section{Experimental design}

The experiment was a completely randomized blocked design with five treatments: (1) inoculation without Funneliformis mosseae (non-AMF); (2) inoculation with F. mosseae only (AMF); (3) inoculation with F. mosseae, in company with exogenous fructose (Fru + AMF); (4) inoculation with F.mosseae, in company with exogenous glucose (Glu + AMF); (5) inoculation with F. mosseae, in company with exogenous sucrose (Suc + AMF). Each treatment replicated four times, resulting in a total of 20 root boxes.

\section{Preparation of two-chambered rootbox}

A rootbox (see Fig. 1 in detail) with $21.5 \mathrm{~cm}$ length, 10 $\mathrm{cm}$ width and $18 \mathrm{~cm}$ height was used in this study and separated into two equal chambers by $37-\mu \mathrm{m}$ nylon mesh, namely, root + hyphae chamber (the presence of roots and mycorrhizal hyphae) and hyphae chamber (the presence of mycorrhizal hyphae or not, free of roots). The 37-um nylon mesh only allows mycorrhizal hyphae pass through from a chamber into another chamber. In addition, an air gap (1.5 $\mathrm{cm}$ width) was created in the center of the rootbox using two layers of 37- $\mu \mathrm{m}$ nylon mesh to reduce the diffusion of substances between the two chambers.

\section{Plant culture}

The AM fungal strain, Funneliformis mosseae (Nicol. \& Gerd.) Schüßler \& Walker [BGC XZ02A], was propagated with the identified fungal spores and white clover (Trifolium repens) for 16 weeks in pots, thereby, containing sands, spores (20 spores/g) and infected root segments.

Seeds of trifoliate orange were sterilized with $70 \%$ alcohol solution for $10 \mathrm{~min}$ and then germinated in autoclaved $\left(0.11 \mathrm{MPa}, 121{ }^{\circ} \mathrm{C}, 2 \mathrm{~h}\right)$ sands under the conditions of $28 / 20{ }^{\circ} \mathrm{C}$ day/night temperature and $80 \%$ relative air humidity. Subsequently, two 5-leaf-old seedlings with uniform size were transplanted into the root + hyphae chamber of the rootbox, in which $1.5 \mathrm{~kg}$ of sterilized $(0.11$ $\mathrm{MPa}, 121^{\circ} \mathrm{C}, 2 \mathrm{~h}$ ) sands and 1000 spores of $F$. mosseae were

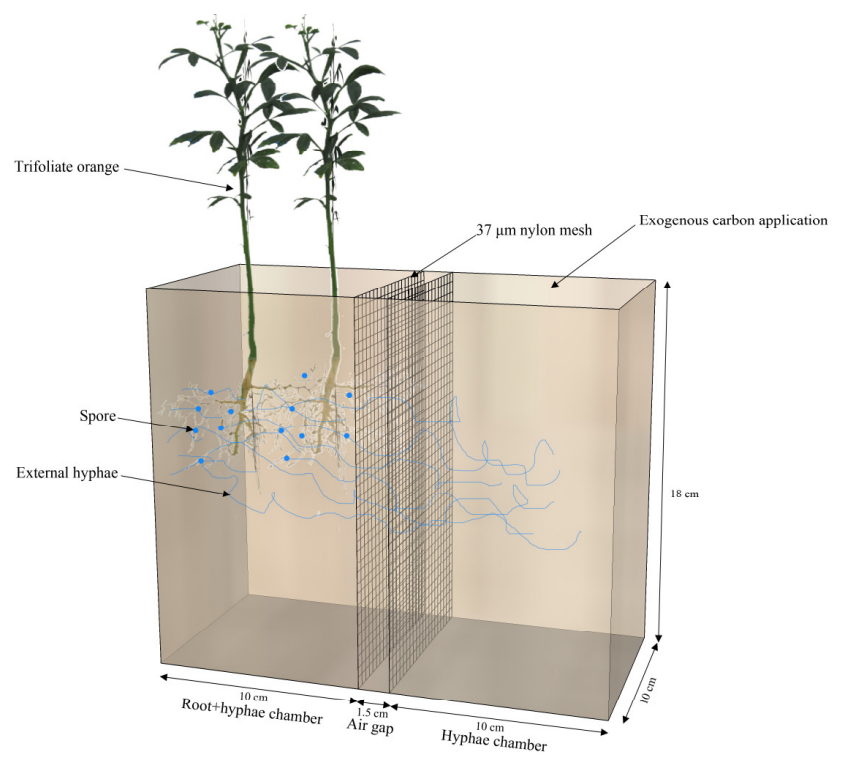

Fig. 1. Schematic representation of a two-chambered rootbox

applied. Simultaneously, hyphae chamber received $1.5 \mathrm{~kg}$ of autoclaved sands and the same amount of autoclaved inoculums. All the rootboxes were placed in a greenhouse of Yangtze University campus, where photon flux density ranged from 721 to $967 \mu \mathrm{mol} / \mathrm{m}^{2} / \mathrm{s}$ with $25 / 19^{\circ} \mathrm{C}$ average day/night temperature and $75-95 \%$ relative air humidity.

During 60 days of AMF inoculation, a $50 \mathrm{~mL}$ Hoagland nutrient solution was irrigated in the root + hyphae chamber of each treatment. And, the hyphae chamber only received $50 \mathrm{~mL}$ distilled water to maintain the substrate moist. Thereafter, $40 \mathrm{mmol} / \mathrm{L}$ fructose, glucose and sucrose were daily supplied into the Hoagland solutions, which collectively applied into the hyphae chamber. Correspondingly, root + hyphae still received $50 \mathrm{~mL}$ Hoagland nutrient solution without exogenous $\mathrm{C}$.

\section{Variable determinations}

Exogenous fructose, glucose and sucrose application was lasted for another 60 days. Subsequently, the seedlings of the root + hyphae chamber were harvested and divided into the shoots and roots. Height, stem diameter, and leaf numbers were measured before plant harvested. The root system of each plant was scanned by the Epson Perfection V700 Photo Dual Lens System (J221A, Indonesia). Root morphological traits, including length, diameter, projected area, surface area, and volume were analyzed with the scanned photo by a WinRHIZO professional software in 2007b (Regent Instruments Inc, Quebec, Canada). Besides, $1-\mathrm{cm}$-long root segments were collected and stained with trypan blue, according to the protocol of Phillips and Hayman (1970). The root mycorrhizal colonization was expressed as the percentage of infected root length against total observed root length.

Sucrose, glucose and fructose concentrations in leaves and roots were determined according to the method of Wu et al. (2010). The activity of acid invertase (AI), neutral invertase (NI), and sucrose synthesis (SS) in synthesis direction and cleavage direction was assayed using the protocol described by Wu et al. (2015). 


\section{Statistical analysis}

Data (means $\pm S D, n=4$ ) were analyzed with ANOVA (SAS, version 8.1), and the significant differences among these treatments were compared with the Fisher's Protected Least Significant Difference (LSD) at $P<0.05$.

\section{Results}

\section{Root mycorrhizal colonization and plant growth}

Root mycorrhizal colonization of trifoliate orange by $F$. mosseae varied from $15.2 \%$ to $39.13 \%$ (Table 1). Exogenous fructose, glucose, and sucrose application into the hyphae chamber significantly increased root mycorrhizal colonization by $155 \%, 133 \%$, and $157 \%$, as compared with sole AMF treatment. Compared with non-AMF treatment, AMF, Fru + AMF, Glu + AMF and Suc + AMF treatments significantly increased plant height by $28 \%, 47 \%$, $42 \%$, and $25 \%$, stem diameter by $23 \%, 20 \%, 10 \%$, and $2 \%$, and leaf number by $13 \%, 33 \%, 29 \%$, and $26 \%$, respectively (Table 1). Moreover, exogenous fructose significantly enlarged positive effects on AMF-induced plant growth traits, as compared with the other $\mathrm{C}$ treatments.

\section{Root morphology}

Compared with non-mycorrhizal treatment, AMF colonization significantly increased root total length, projected area, surface area, volume, and average diameter of trifoliate orange seedlings, irrespective of exogenous $\mathrm{C}$ applied or not (Table 1). Besides, compared with sole AMF treatment, exogenous $\mathrm{C}$ application further significantly increased root projected area, surface area, volume, and average diameter by $10 \%, 10 \%, 12 \%$, and $6 \%$ under Fru + AMF conditions, $10 \%, 10 \%, 12 \%$, and $10 \%$ under Glu + AMF conditions, and 3\%, 3\%, 9\%, and $8 \%$ under Suc + AMF conditions, respectively.

\section{Carbohydrate concentrations in leaf and root}

In comparison with non-mycorrhizal treatment, AMF colonization dramatically promoted carbohydrate concentrations of trifoliate orange seedlings, in spite of exogenous $\mathrm{C}$ supplied or not (Fig. 2a-c). Compared with sole AMF treatment, sucrose contents decreased significantly by $23 \%, 26 \%$, and $16 \%$ in leaves under Fru + AMF, Glu + AMF, and Suc + AMF conditions, while increased by $2 \%, 14 \%$ and $27 \%$ in roots, respectively (Fig. 2a). Leaf glucose contents increased significantly by $16 \%$ under Fru + AMF treatment compared with sole AMF treatment, while decreased dramatically by $12 \%$ and $18 \%$ under Glu + AMF and Suc + AMF treatments, respectively (Fig. 2b). Furthermore, root glucose contents were on a rise, except the exogenous sucrose treatment, under which root glucose contents decreased instinctively by $2 \%$ as compared with sole AMF treatment. Besides, compared with sole AMF treatment, leaf fructose contents increased prominently by $15 \%$ under Fru + AMF treatment, while decreased significantly by $20 \%$ and $33 \%$ under Glu + AMF and Suc + AMF treatments, respectively (Fig. 2c). And, in comparison with sole AMF treatment, root fructose contents promoted significantly by $11 \%$ and $27 \%$ under Fru + AMF and Glu + AMF conditions, while decreased observably by $10 \%$ under Suc + AMF treatment.

\section{Activities of sucrose metabolic enzymes in leaf and root}

Compared with non-AMF treatment, sole AMF treatment significantly affected SS activities: $39 \%$ and 36\% higher in leaf synthesis and root cleavage directions; 39\% and 203\% lower in root synthesis and leaf cleavage directions (Fig. 3a, 3b). Compared with sole AMF treatment, leaf SS activity reduced dramatically by $76 \%$, $45 \%$, and $44 \%$ in synthesis direction with exogenous fructose, glucose and sucrose application, while increased significantly by $396 \%$ and $272 \%$ in cleavage direction with exogenous fructose and glucose, except Suc + AMF treatment. In comparison with sole AMF treatment, root SS activity represented a rising trend by exogenous C treatments regardless of synthesis direction and cleavage direction, except Fru + AMF treatment (a 15\% lower root SS activity in synthesis direction).

Compared with non-AMF treatment, alone AMF seedlings had $75 \%$ and $13 \%$ higher leaf and root AI activity (Fig. 4a). However, exogenous $\mathrm{C}$ application dramatically reduced leaf AI activities as compared with sole AMF treatment: 52\%, 61\%, and $72 \%$ lower under Fru + AMF, Glu + AMF, and Suc + AMF conditions, respectively. Compared with sole AMF treatment, root AI activity increased significantly by $45 \%$ with the supplication of exogenous fructose, while decreased evidently by $26 \%$ with the addition of exogenous glucose. No significant difference in root AI activity was observed between alone AMF and Suc + AMF treatment.

Sole AMF seedlings possessed $13 \%$ and $72 \%$ higher significantly leaf and root $\mathrm{NI}$ activity compared with nonAMF controls (Fig. 4b). Moreover, Glu + AMF and Suc +

Table 1. Effects of exogenous fructose, glucose, and sucrose application into hyphae chamber on root mycorrhizal colonization, plant growth, and root morphological traits of Funneliformis mosseae-colonized trifoliate orange (Poncirus trifoliata) seedlings grown in a two-chambered rootbox

\begin{tabular}{|c|c|c|c|c|c|c|c|c|c|}
\hline \multirow[b]{2}{*}{ Treatments } & \multirow{2}{*}{$\begin{array}{c}\text { Root } \\
\text { mycorrhizal } \\
\text { colonization } \\
(\%)\end{array}$} & \multicolumn{3}{|c|}{ Plant growth performance } & \multicolumn{5}{|c|}{ Root morphological traits } \\
\hline & & $\begin{array}{l}\text { Plant } \\
\text { height } \\
(\mathrm{cm})\end{array}$ & $\begin{array}{c}\text { Stem } \\
\text { diameter } \\
(\mathrm{mm})\end{array}$ & $\begin{array}{c}\text { Leaf } \\
\text { number } \\
(\# / \text { plant })\end{array}$ & $\begin{array}{c}\text { Total length } \\
(\mathrm{cm})\end{array}$ & $\begin{array}{c}\text { Projected } \\
\text { area } \\
\left(\mathrm{cm}^{2)}\right.\end{array}$ & $\begin{array}{l}\text { Surface area } \\
\left(\mathrm{cm}^{2)}\right.\end{array}$ & $\begin{array}{l}\text { Volume } \\
\left(\mathrm{cm}^{3}\right)\end{array}$ & $\begin{array}{c}\text { Average } \\
\text { diameter } \\
(\mathrm{mm})\end{array}$ \\
\hline non-AMF & $0 \pm 0 \mathrm{~d}$ & $19.6 \pm 1.6 \mathrm{c}$ & $2.81 \pm 0.15 \mathrm{c}$ & $22 \pm 1 c$ & $153 \pm 16 c$ & $16.5 \pm 0.6 c$ & $51.7 \pm 4.0 \mathrm{c}$ & $1.40 \pm 0.05 c$ & $1.07 \pm 0.03 c$ \\
\hline AMF & $15.20 \pm 0.63 \mathrm{c}$ & $25.2 \pm 1.9 \mathrm{~b}$ & $3.46 \pm 0.08 \mathrm{a}$ & $25 \pm 1 b$ & $182 \pm 20 b$ & $20.5 \pm 0.7 b$ & $64.5 \pm 2.4 b$ & $1.87 \pm 0.03 \mathrm{~b}$ & $1.10 \pm 0.08 \mathrm{bc}$ \\
\hline Fru + AMF & $38.73 \pm 1.94 a$ & $28.9 \pm 1.8 \mathrm{a}$ & $3.37 \pm 0.09 \mathrm{a}$ & $29 \pm 1 a$ & $197 \pm 12 \mathrm{a}$ & $22.6 \pm 0.8 a$ & $71.0 \pm 4.0 \mathrm{a}$ & $2.10 \pm 0.07 a$ & $1.17 \pm 0.05 \mathrm{ab}$ \\
\hline Glu + AMF & $35.40 \pm 2.74 b$ & $27.8 \pm 1.0 \mathrm{a}$ & $3.09 \pm 0.09 \mathrm{~b}$ & $29 \pm 2 \mathrm{a}$ & $191 \pm 15 \mathrm{ab}$ & $22.6 \pm 0.6 a$ & $71.1 \pm 6.0 \mathrm{a}$ & $2.11 \pm 0.06 \mathrm{a}$ & $1.21 \pm 0.07 \mathrm{a}$ \\
\hline Suc + AMF & $39.13 \pm 1.16 a$ & $24.5 \pm 1.5 b$ & $2.88 \pm 0.22 \mathrm{c}$ & $28 \pm 2 a$ & $189 \pm 10 \mathrm{ab}$ & $21.2 \pm 0.6 \mathrm{ab}$ & $66.5 \pm 3.5 \mathrm{ab}$ & $2.04 \pm 0.03 a$ & $1.19 \pm 0.05 \mathrm{a}$ \\
\hline
\end{tabular}

Data (means \pm SD, $n=4)$ followed by different letters in a row indicate significant differences $(P<0.05)$ between treatments 


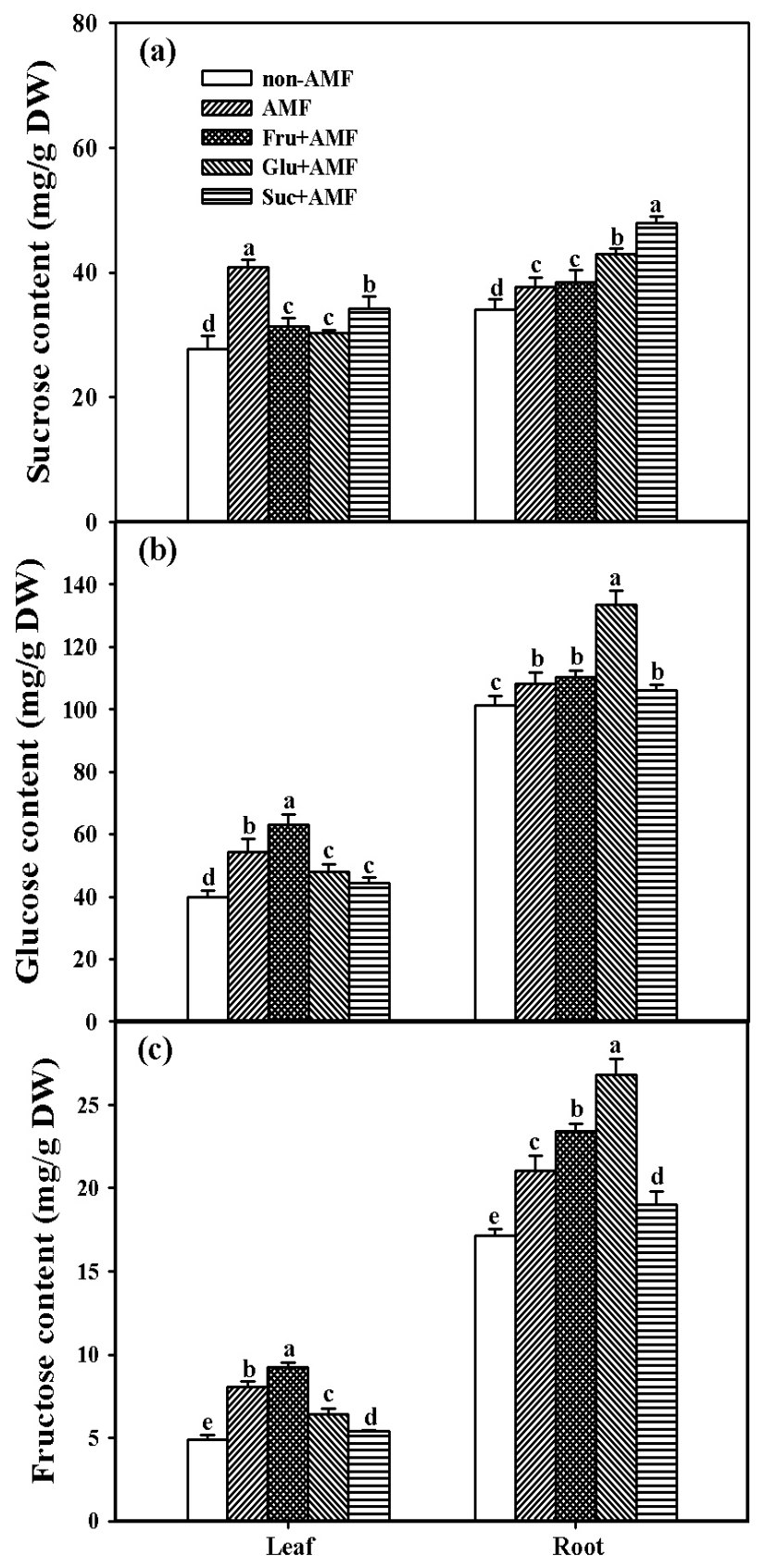

Fig. 2. Effects of exogenous fructose, glucose, and sucrose application into hyphae chamber on sucrose (a), glucose (b), and fructose (c) concentrations in leaf and root of Funneliformis mosseae-colonized trifoliate orange (Poncirus trifoliata) seedlings grown in a two-chambered rootbox. Data (means $\pm \mathrm{SD}, n=4)$ are significantly different $(P<0.05)$ followed by different letters above the bars

AMF treatments prominently decreased leaf NI activity by $20 \%$ and $27 \%$ in comparison with sole AMF treatment, except Fru + AMF treatment. Furthermore, under mycorrhization, root NI activity increased evidently by $32 \%$ and $8 \%$ with the presence of exogenous fructose and sucrose, while decreased dramatically by $50 \%$ with exogenous glucose supply.

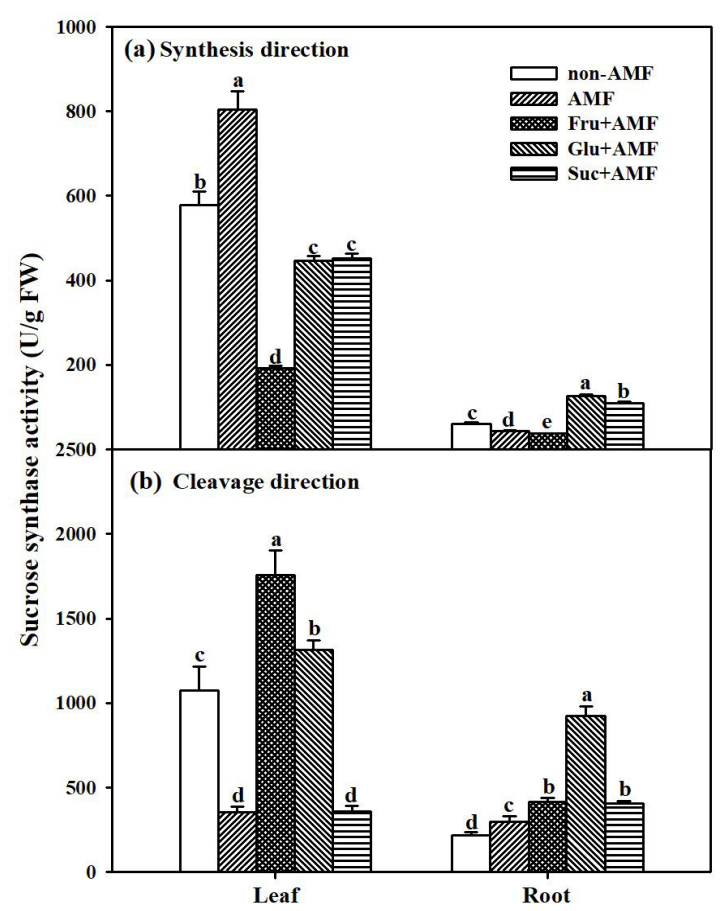

Fig. 3. Effects of exogenous fructose, glucose, and sucrose application into hyphae chamber on sucrose synthase activities in synthesis direction (a) and cleavage direction (b) in leaf and root of Funneliformis mosseae-colonized trifoliate orange (Poncirus trifoliata) seedlings grown in a two-chambered rootbox. Data (means $\pm \mathrm{SD}, n=4$ ) are significantly different $(P<0.05)$ followed by different letters above the bars

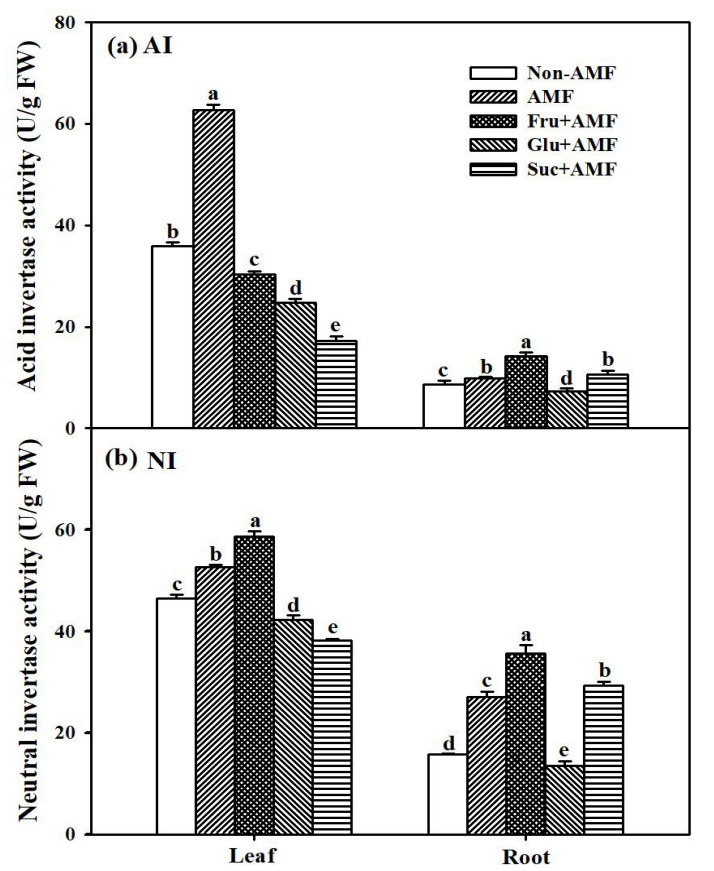

Fig. 4. Effects of exogenous fructose, glucose, and sucrose application into hyphae chamber on sucrose synthase activities in synthesis direction (a) and cleavage direction (b) in leaf and root of Funneliformis mosseae-colonized trifoliate orange (Poncirus trifoliata) seedlings grown in a two-chambered rootbox. Data (means $\pm \mathrm{SD}, n=4$ ) are significantly different $(P<0.05)$ followed by different letters above the bars 


\section{Discussion}

In this work, root mycorrhizal colonization of trifoliate orange by $F$. mosseae could be heavily stimulated by exogenous fructose, glucose, and sucrose application into the hyphae chamber. Meanwhile, fructose and sucrose showed better effect than glucose. This indicated that C source could be assimilated by external hyphae in the hyphae chamber, resulting in the positive effect on growth and propagation of AMF. Earlier study by Bécard et al. (1989) showed that C not only accelerated spore germination, but also facilitated hyphae growth. Therefore, it could be speculated that the response of root colonization to exogenous $\mathrm{C}$ might be attributed to spore behaviour.

In this study, sole AMF inoculation significantly improved plant growth performance of trifoliate orange seedlings. And, exogenous application of carbohydrates, especially fructose, further magnified AMF effects on improved plant growth. This manifested that the carbon absorbed by mycorrhizal hyphae was aptly used for mycorrhizal plant growth. Lastdrager et al. (2014) reported that sugars have a central regulatory function in steering plant growth through the regulation of protein synthesis. Moreover, sugars can generally act as signalling molecules cross-talk with various hormones to modulate critical aspects of plant growth through signalling pathways (Eveland and Jackson, 2011).

In the present work, alone AMF inoculation induced higher root morphological traits than non-inoculation treatment, which was in line with the result reported by Wu et al. (2011). Moreover, exogenous $C$ treatments under mycorrhization maintained a significantly higher magnitude of root morphological characters than sole mycorrhizal treatment. This demonstrated that $\mathrm{C}$ source assimilated by external hyphae markedly promoted AMF roles in improving the root morphology. Meanwhile, exogenous fructose exhibited the best positive effects on AMFimproved root morphology. As a result, exogenous C, especially fructose, will enlarge AMF effects on improved root morphology of trifoliate orange, which is important for water and nutrient absorption. However, Jiang et al. (2017) reported that the fatty acids synthesized in the host plants, but not sugars, were a major source of organic $\mathrm{C}$ delivered to fungus for sustaining mycorrhizal colonization, indicating that fatty acids were necessary for AMs. It concludes that fatty acids might deliver more easily between host plants and AM fungus, and exogenous $\mathrm{C}$, especially fructose, is more easy to induce the production of fatty acids for plant growth. In this regard, more works will be conducted.

Spore germination of AMF depends on host photosynthates (Bago et al., 2003). The present work indicated that sole AMF treatment strongly stimulated the production of sucrose, glucose and fructose contents in leaf and root, in comparison with non-AMF treatment, which is in accordance with $\mathrm{Wu}$ et al. (2014). In addition, mycorrhizal seedlings treated with exogenous $\mathrm{C}$ induced higher carbohydrate concentrations in root and lower carbohydrate concentrations in leaf than sole mycorrhizal seedlings without exogenous $C$. It seems that more sucrose was transported from source leaves into roots for cleavage. However, Baier et al. (2010) reported that AMF inoculation down-regulated $S S$ gene expression in roots of an AMF-plant system, which is contrary to the present results. It may be because a great deal of carbohydrates accumulates in AM roots after the participation of exogenous $\mathrm{C}$, and thus the increase in root SS (cleavage direction) activity dramatically ensures sucrose cleavage for AM and root growth. Xie et al. (2013) reported the competitive relation between AMF and the host plants on photosynthates to a certain degree.

Sucrose synthase (SS) is divided into the synthesis direction and cleavage direction, and collectively catalyzes the reversible reaction of sucrose into hexoses (Verma et al., 2013). The present study indicated that AMF colonization significantly increased leaf SS (synthesis direction) activity than non-AMF treatment, confirming that AMs induced higher SS (synthesis direction) activity to synthesize sucrose in leaves. Earlier evidence also confirmed that the photosynthetic rate of the host plant was enhanced by mycorrhizas to meet the increased carbohydrate requirement for AMs (Ferrol et al., 2009). Moreover, the decline of the leaf SS (synthesis direction) activity was in line with the decrease of leaf sucrose content in mycorrhizal seedlings. This may be due to abundant $\mathrm{C}$ sources in mycorrhizosphere, and there is no need to consume energy inside plants for accelerating the functions of SS after the participation of sufficient exogenous C. Additionally, the increase in root SS (cleavage direction) activity maintained the correspondence with the increase of root glucose and fructose contents in mycorrhizal seedlings with exogenous carbon compared with non-AMF treatment. As reported by Bago et al. (2003), AMF could only assimilate micromolecule hexose, and thus, C sources absorbed by external hyphae needed to be broken down into glucose and fructose, which need the increase of root SS (cleavage direction) activity.

Plant invertases primarily catalyze the irreversible hydrolysis of sucrose into glucose and fructose (Moscatello et al., 2011). In our study, alone AMF inoculation dramatically improved AI and NI activities in both leaves and roots, indicating that mycorrhizal plants need more hexoses for AM growth and development in terms of sucrose-cleaving enzymes (Hodge et al., 2001). In addition, exogenous glucose prominently weakened mycorrhizal effects on the enhancement of invertase activities, indicating that there is no need to consume energy for accelerating the functions of $\mathrm{AI}$ and $\mathrm{NI}$ after the participation of sufficient exogenous glucose (Bago et al., 2003). In AM plants, exogenous fructose supplication showed diverse changes in leaf and root: decrease of leaf $\mathrm{AI}$ and increase of root $\mathrm{AI}$ activity; increase of leaf and root NI activity. As reported by Lewis and Harley (1965), exogenous glucose principally induced the accumulation of trehalose and glycogen, and fructose modulated the accumulation of mannitol. It seems that in citrus mycorrhizas, mannitol is possibly more important than trehalose and glycogen in AM development.

\section{Conclusions}

Inoculation with $F$. mosseae significantly improved plant growth performance and root morphological traits of trifoliate orange, which could be further promoted by exogenous $\mathrm{C}$ applied into hyphae chamber, especially fructose. 
370

\section{Acknowledgements}

This study was supported by the Plan in Scientific and Technological Innovation Team of Outstanding Young, Hubei Provincial Department of Education (T201604).

\section{References}

Bago B, Pfeffer PE, Shachar-Hill Y (2000). Carbon metabolism and transport in arbuscular mycorrhizas. Plant Physiology 124(3):949-958.

Bago B, Zipfel W, Williams RM, Jun J, Arreola R, Lammers PJ, ... ShacharHill Y (2002). Translocation and utilization of fungal storage lipid in the arbuscular mycorrhizal symbiosis. Plant Physiology 128(1):108-124.

Bago B, Pfeffer PE, AbubakerJ, Jun J, Allen JW, Brouillette J,... Shachar-Hill Y (2003). Carbon export from arbuscular mycorrhizal roots involves the translocation of carbohydrate as well as lipid. Plant Physiology 131(3):1496-1507.

Baier MC, Keck M, Godde V, Niehaus K, Kuster H, Hohnjec N (2010). Knockdown of the symbiotic sucrose synthase MtSucS1 affects arbuscule maturation and maintenance in mycorrhizal roots of Medicago truncatula. Plant Physiology 152(2):1000-1014.

Bécard G, Piché Y (1989). Fungal growth stimulation by $\mathrm{CO}_{2}$ and root exudates in vesicular-arbuscular mycorrhizal symbiosis. Applied and Environmental Microbiology 55:2320-2325.

Eveland AL, Jackson DP (2011). Sugars, signalling, and plant development.Journal of Experimental Botany 63(9):3367-3377.

Ferrol N, Pérez-Tienda J (2009). Coordinated nutrient exchange in arbuscular mycorrhiza. In: Azcón-Aguilar C, Barea JM, Gianinazzi S, Gianinazzi-Pearson V (Eds). Mycorrhizas-Functional Processes and Ecological Impact. Springer, Berlin Heidelberg pp 73-87.

Gadkar V, David-Schwartz R, Kunik T, Kapulnik Y (2001). Arbuscular mycorrhizal fungi colonization. Factors involved in host recognition. Plant Physiology 127(4):1493-1499.

HeZQ, He CX, Zhang ZB, Zou RZ, Wang HS (2006). Effects of various arbuscular mycorrhizal fungi on tomato growth and related physiological factors. Journal of Shenyang Agricultural University 37(3):308-312 (in Chinese with English abstract).

Hodge A, Campbell C, Fitter AH (2001). An arbuscular mycorrhizal fungus accelerates decomposition and acquisition nitrogen directly from organic material. Nature 413:297-299.

Jiang Y, Wang W, Xie Q, Liu N, Liu L, Wang D, ... WangE (2017). Plants transfer lipids to sustain colonization by mutualistic mycorrhizal and parasitic fungi. Science 356(6343):1172-1175.

Jin HR, Jiang DH, Zhang PH (2011). Effect of carbon and nitrogen availability on metabolism of amino acids in germinating spores of arbuscular mycorrhizal fungi. Pedosphere 21(4):432-442.

Lastdrager J, Hanson J, Smeekens S (2014). Sugar signals and the control of plant growth and development. Journal of Experimental Botany 65(3):799-807.
Lewis DH, HarleyJL (1965). Carbohydrate physiology of mycorrhizal roots of beech. New Phytologist 64(2):224237.

Luginbuehl LH, Menard GN, Kurup S, Van Erp H, Radhakrishnan GV, Breakspear A, ... Eastmond PJ (2017). Fatty acids in arbuscular mycorrhizal fungi are synthesized by the host plant. Science 356(6343):1175-1178.

Moscatello S, Famiani F, Proietti S, Farinelli D, Battistelli A (2011). Sucrose synthase dominates carbohydrate metabolism and relative growth rate in growing kiwifruit (Actinidia deliciosa, ov Hayward).Scientia Horticulturae 128(3):197-205.

O'Hara LE, Paul MJ, Wingler A (2013). How do sugars regulate plant growth and development? New insight into the role of trehalose- 6 phosphate. Molecular Plant 6(2):261-274.

Phillips JM, Hayman DS (1970). Improved procedures for clearing roots and staining parasitic and vesicular-arbuscular mycorrhizal fungi for rapid assessment of infection. Transactions of the British Mycological Society 55(1):158-161.

Schubert A, Allara P, Morte A (2004). Cleavage of sucrose in roots of soybean (Glycine max) colonized by an arbuscular mycorrhizal fungus. New Phytologist 161(2):495-501.

Verma AK, Agarwal AK, Dubey RS, Solomon S, Singh SB (2013). Sugar partitioning in sprouting lateral bud and shoot development of sugarcane. Plant Physiology and Biochemistry 62:111-115.

Wu QS, Peng YH, Zou YN, Liu CY (2010). Exogenous polyamines affect mycorrhizal development of Glomus mosseae colonized citrus (Citrus tangerine) seedlings. ScienceAsia 36:254258.

Wu QS, Srivastava AK, Li Y (2015). Effect of mycorrhizal symbiosis on growth behavior and carbohdyrate metabolism of trifoliate orange under different substrate P levels. Journal of Plant Growth Regulation 34:495-508.

Wu QS,Zou YN,HeXH,LuoP (2011). Arbuscular mycorrhizal fungi can alter some root characters and physiological status in trifoliate orange (Poncirus trifoliata L. Raf.) seedlings. Plant Growth Regulation 65:273278.

Wu QS, Zou YN, Huang YM, Li Y, He XH (2013). Arbuscular mycorrhizal fungi induce sucrose cleavage for carbon supply of arbuscular mycorrhizas in citrus genotypes. Scientia Horticulturae 160:320-325.

Wu QS, Yuan FY, Fei YJ, Li L, Huang YM, Liu CY (2014). Effects of arbuscular mycorrhizal fungi on root system architecture and sugar contents of white clover. Acta Prataculturae Sinica 23(1):199-204.

Xie XI, Gu ZH, Zhu HH, Yao Q (2013). Correlation between glomalin related soil protein and root morphology. Mycosystema 32:993-1003 (in Chinese with English abstract).

Zobel RW, Alloush GA, Belesky DP (2006). Differential root morphology response to no versus high phosphorus, in three hydroponically grown forage chicory cultivars. Environmental and Experimental Botany 57(1):201-208. 